\title{
Seeing topological edge and bulk currents in time-of-flight images
}

\author{
Alvaro Rubio-García $\odot,{ }^{1,{ }^{*}}$ Chris N. Self,,${ }^{2,}$ Juan Jose García-Ripoll $\odot,{ }^{3}$ and Jiannis K. Pachos $\odot^{2}$ \\ ${ }^{1}$ Instituto de Estructura de la Materia IEM-CSIC, Calle Serrano 123, Madrid E-28006, Spain \\ ${ }^{2}$ School of Physics and Astronomy, University of Leeds, Leeds LS2 9JT, United Kingdom \\ ${ }^{3}$ Instituto de Física Fundamental IFF-CSIC, Calle Serrano 113b, Madrid E-28006, Spain
}

(Received 13 October 2019; accepted 14 July 2020; published 23 July 2020)

\begin{abstract}
Here we provide a general methodology to directly measure the topological currents emerging in the optical lattice implementation of the Haldane model. Alongside the edge currents supported by gapless edge states, transverse currents can emerge in the bulk of the system whenever the local potential is varied in space, even if it does not cause a phase transition. In optical lattice implementations the overall harmonic potential that traps the atoms provides the boundaries of the topological phase that supports the edge currents, as well as providing the potential gradient across the topological phase that gives rise to the bulk current. Both the edge and bulk currents are resilient to several experimental parameters such as trapping potential, temperature, and disorder. We propose to investigate the properties of these currents directly from time-of-flight images with both short-time and long-time expansions.
\end{abstract}

DOI: 10.1103/PhysRevB.102.041123

Introduction. Two-dimensional Chern insulators are described by a nonzero integer topological index, typically given by the Chern number, $v$ [1-5]. At physical boundaries of the system the Chern number changes from a nonzero value inside the material to a zero value outside it. This change can be interpreted as a topological phase transition, which is manifested at the boundary of the topological system as a one-dimensional gapless edge state [1-6]. As the change in the Chern number necessarily takes only integer values rather than the continuum the edge states are thus robust to small perturbations and finite temperature. In the presence of a constant chemical potential $V(\boldsymbol{r})=V$ the particle-hole imbalance in the population of the edge states gives rise to the edge current

$$
I_{\text {edge }}=\frac{\nu V}{2 \pi},
$$

traversing along the boundary of the system [7-10]. The edge currents provide a powerful tool to experimentally probe the topological properties of the system.

Alongside the edge currents, the bulk of the Chern insulator can support currents, whenever the local potential has a nonzero gradient [10-12], as could happen if the system was placed inside an electric field $E$. In contrast to the edge currents, these bulk currents are not activated by moving the Fermi level so that it occupies low-energy orbitals, such as gapless modes. In our previous paper [10] we showed that the application of a potential gradient in the bulk of a Chern insulator produces a set of localized bulk states in the valence and conduction bands, away from the edge modes. Furthermore, they are localized along the potential gradient and produce a current in the direction perpendicular to the gradient. The emergence of these localized states is easier to

${ }^{*}$ These authors contributed equally to this work. explain for sharp potential gradients, such as a potential step. In that case, the gradient divides the system into two regions with different potentials and thus the system eigenmodes tend to be either delocalized inside regions with the same potential or localized in the boundary between these two regions. Moreover, one could picture these localized states as if the carried two chiral opposite currents that cancel each other in the vanishing gradient limit, as their population is balanced. For small nonzero gradients, below the energy gap, this population balance is broken and one current dominates over the other, thus giving rise to the topological bulk current along the potential gradient. For more details, we refer the reader to Ref. [10]. The intensity of these currents is given by

$$
I_{\text {bulk }}(\boldsymbol{r})=\frac{v}{2 \pi} a_{0}|\nabla V(\boldsymbol{r})|,
$$

with $a_{0}$ the lattice constant along the direction of the potential gradient. Same as the edge currents, the bulk currents are also quantized by the Chern number $v$. In the case in which the potential gradient was caused by a general electric field, then the bulk current intensity would be proportional to the field $I_{\text {bulk }}(\boldsymbol{r}) \propto v E(\boldsymbol{r})$. The potential must vary on the scale of $a_{0}$ for bulk currents to flow. This is equivalent to the edge currents in a discrete lattice system, where the chemical potential must be varied by more than the finite size energy spacing of states in order to observe edge currents. Similar to the edge currents the bulk currents are robust against temperature and local disorder [10]. In the case in which there is a net potential gradient, the edge currents traveling along the system boundaries are

$$
I_{\text {edge }}(\boldsymbol{r})=\frac{\nu V(\boldsymbol{r})}{2 \pi} .
$$

Unlike the edge currents the position and direction of the topological bulk currents can be controlled at will by external potentials. Hence, they offer a unique platform for developing new technologies from transport currents that are directional 
and frictionless to programmable transistors, amplifiers, and detectors [13-17].

A physical system where edge and bulk currents can naturally emerge is optical lattice implementations of the Haldane model [18-22]. Time-of-flight images enable the measurement of the Chern number of this system thus allowing us to confirm its topological nature [23]. In these experiments harmonic potentials are used to keep the atoms localized in a certain region, in addition to the Hamiltonian of the Haldane model. This trapping causes a spatial variation in the local potential. The effects of this smoothly varying potential on the topological edge modes of the system have been well studied $[6,24]$. Here we demonstrate that the variation of the trapping potential across the bulk topological phase also gives rise to interesting topological effects in the bulk, specifically to protected bulk currents. Furthermore, the interplay between edge and bulk currents can be obtained by time-of-flight (TOF) images making their investigation directly accessible to current experiments. We find signatures of the edge and bulk currents in both the short-time and long-time expansion images, thus offering novel and versatile means of probing topological current physics.

Realizing topological edge and bulk currents in optical lattices. To investigate the properties of the topological edge and bulk currents we consider the implementation of the Haldane model with ultracold atoms in optical lattices. The Haldane model is defined on a hexagonal lattice with a fermionic mode $\left\{c_{i}, c_{i}^{\dagger}\right\}$ living at each vertex of the lattice. The Hamiltonian is

$$
H=\sum_{\langle i j\rangle} t_{1} c_{i}^{\dagger} c_{j}+\sum_{\langle\langle i j\rangle\rangle} t_{2} e^{i v_{i j} \phi} c_{i}^{\dagger} c_{j}+\sum_{i} V\left(\boldsymbol{r}_{i}\right) c_{i}^{\dagger} c_{i},
$$

where $t_{1}$ and $t_{2}$ are the nearest- and next-nearest-neighbor hopping strengths, respectively, $\phi$ is a complex phase, $v_{i j}$ a parameter that is \pm 1 depending on the direction of the hopping $i \rightarrow j$, as shown in Fig. 1, and $V(r)$ is a local potential. For certain choices of parameters the Haldane model has a topological phase with Chern number $v= \pm 1$ as well as a trivial phase where $v=0$ [25]. All results here are produced for the parameters $t_{1}=1, t_{2}=0.1, \phi=\pi / 2$ that, at halffilling and for $V(\boldsymbol{r})=0$ give $v=1$ [23].

Motivated by initial theoretical work [23], several experimental realizations of the Haldane model in optical lattices have been achieved [18-22]. In these experiments the Haldane lattice model given by (4) is engineered inside a harmonic potential trap that keeps the atoms localized in a certain region. We describe the harmonic trap as

$$
V(r)=V_{0}+k\left(\frac{r}{r_{\max }}\right)^{2},
$$

where $V_{0}$ is an overall chemical potential, while $k$ and $r_{\max }$ are suitable parameters of the trap. Depending on the choice of the constants $V_{0}$ and $k$ this potential can give rise to a "wedding cake" structure, with three phases emerging at different radii from the center of the harmonic trap, as shown in Fig. 1. Near the center, $r=0$, a trivial phase is nested with a lattice filling fraction given by $n=1$ and local Chern number $v=0$ as all bands of the system are populated (we assume the local density approximation is valid for broad enough traps as the ones we used in our simulations so that the Chern number is

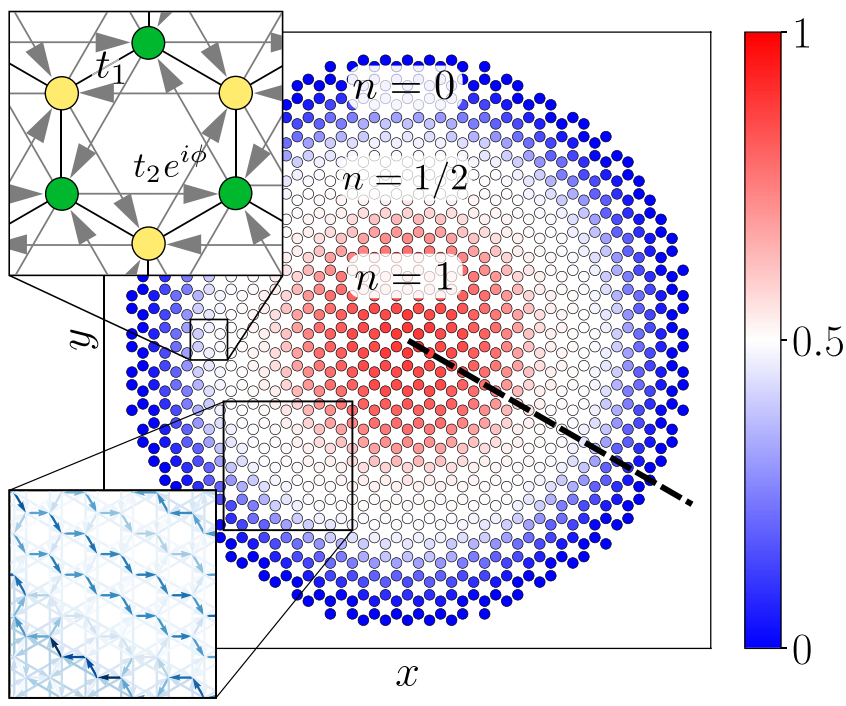

FIG. 1. Real-space occupations of the Haldane model inside a harmonic trap. There are three clearly visible phases with densities $n=1$ (red), $n=1 / 2$ (white), and $n=0$ (blue). We are interested in the flux of the currents crossing the radial dashed line. Upper detail: Hexagonal plaquette of the Haldane lattice model. Arrows denote hopping directions for which $v_{i j}=+1$ in Eq. (4). Lower detail: Realspace currents (arrows) between lattice sites. Intensity of the arrow color denotes the intensity of the current. Parallel edge currents are visible at the boundary of the topological phase, while the current in the bulk has the opposite orientation. Data shown is for $T=0$ and a lattice with 1200 sites. The harmonic potential (5) has $V_{0}=-2.1$, $k=5.6$, and $r_{\max }=13 \sqrt{3}$.

$v= \pm 1$ whenever the local on-site potential falls inside the energy gap). Next an annulus emerges with half-filling, $n=$ $1 / 2$, that corresponds to the Haldane phase with Chern number $v= \pm 1$. Finally, far from the center, where the harmonic potential is too large, a trivial $v=0$ phase is present due to zero band population, $n=0$. For the system sizes we study only the $n=1 / 2$ phase appears to plateau; however, a stable $n=1$ phase would also be present for larger systems [23]. The topologically nontrivial annulus configuration has two boundaries neighboring the trivial $n=1$ and $n=0$ phases. Moreover, the trapping potential varies radially across the topological phase, as seen in Fig. 2. Hence, due to (1) and (2) we expect edge and bulk currents to naturally emerge without additional engineering of the system.

The density current, $J_{i j}$, flowing between two sites $i$ and $j$ of the lattice is dictated by the continuity equation for single site occupation [10]:

$$
J_{i j}=2 \operatorname{Im}\left\{A_{i j}\left\langle c_{i}^{\dagger} c_{j}\right\rangle\right\}
$$

with $A$ the Hamiltonian matrix containing the hopping terms and on-site potentials. From that we can determine the distribution of the current flowing across a cross section of the lattice that goes radially from the center of the trap, as shown in Fig. 1. The local profile of currents supported in the system is shown as a histogram in Fig. 2. We observe that as the harmonic potential has a smooth profile the edge states expand over a large range of the system. Moreover, the smooth change 


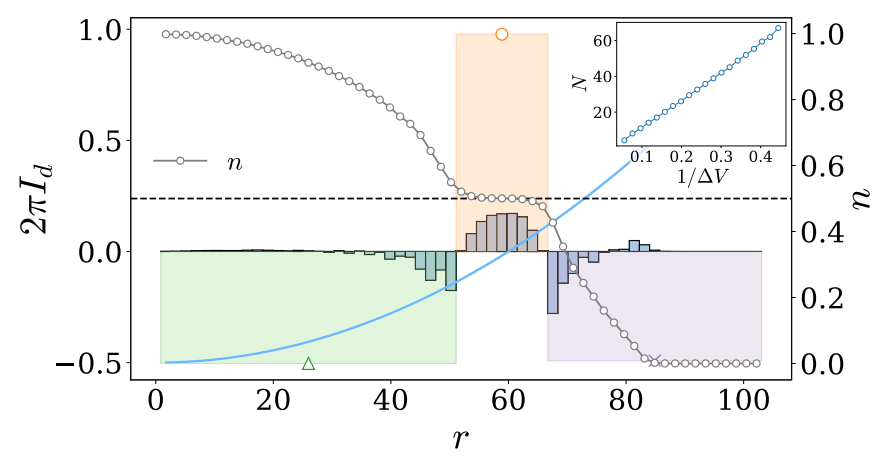

FIG. 2. Occupation of lattice sites (circles) along a radial line (as shown in Fig. 1) inside a harmonic potential trap (blue line), and local currents across small bins (bars) in the same radial line, with $r$ the distance from the center of the trap. Three phases are clearly visible from the occupations: $n=1$ at the center of the trap, $n=1 / 2$ in the middle corresponding to an annulus of the Haldane phase, and $n=0$ at the far end of the system. The total edge and bulk currents resulting from the sum of the small bins are indicated with the three large bars. The total edge currents are equal in magnitude, propagating in the same direction (negative value), while the bulk current through the $n=1 / 2$ region is opposite (positive value) with double magnitude. Data presented is for $T=0$ and a lattice size of roughly 28800 sites. The harmonic potential (5) has $V_{0}=-3, k=9$, and $r_{\max }=60 \sqrt{3}$. Inset: The width $N$ of the topological phase increases linearly with $1 / \Delta V$, where $\Delta V$ is the potential difference at the two boundaries of the phase.

of the local potential in the bulk causes the bulk currents to disperse over the whole topological phase.

In Fig. 2 we also plot the total edge and bulk currents as the sum of the local ones; it is clear that the two edge currents are equal and flowing in the same direction while the bulk current is twice their value and flowing in the opposite direction. This behavior is expected from assuming that the topological phase is stable in the middle of the bulk, where the local potential favoring half-filling $n=1 / 2$, while the potential increases as we move further out and decreases as we move closer to the center of the trap. In this case two opposite potentials are formed at the inner and outer boundaries of the system causing the parallel edge currents traversing along the same direction due to (3). At the same time the linear interpolation of the potential between the two boundaries causes a bulk current to be formed as dictated by (2). This bulk current is opposite to the edge currents in direction but has the same magnitude, because

$$
\sum_{i:\left\{n_{i}\right\rangle=1 / 2} a_{0}\left|\nabla V\left(\boldsymbol{r}_{i}\right)\right| \approx\left|V\left(\boldsymbol{r}_{\text {outer }}\right)-V\left(\boldsymbol{r}_{\text {inner }}\right)\right| .
$$

Therefore, the sum of the bulk and edge currents is zero. Finally, we observe in Fig. 2 (inset) that the width, $N$, of the Haldane phase increases inversely proportional to the potential difference, $\Delta V$, between the two boundaries. This possible control in the macroscopic properties of the system can facilitate the manipulation of the density and spatial extension of the currents under investigation in order to improve their visibility.

Detecting density currents with time-of-flight images. The density currents of the atoms in the $n=1 / 2$ phase of the

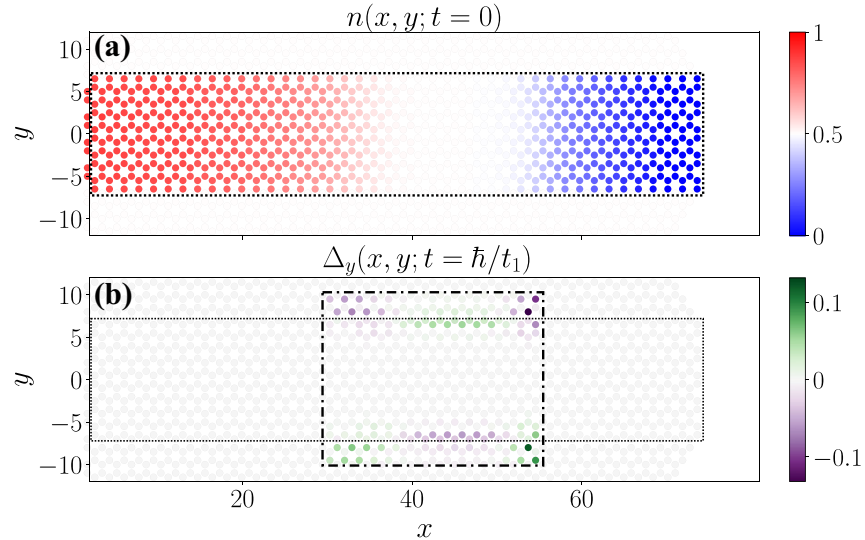

FIG. 3. (a) Densities of a sample of sites (inside dashed line) at a stationary state inside a harmonic trap isolated by a mask. Densities outside the sample are taken to be 0. (b) Relative density difference $\Delta_{y}(x, y ; t)$ inside a smaller region (dot-dashed line) after a short-time expansion $\left(t=\hbar / t_{1}\right)$ of the sites within the initial sample when the trap is turned off. A clear pattern of density imbalances is already observed, that corresponds to one up-going current inside the Haldane phase and two down-going currents at its boundaries. Data shown is for $T=0$ and a lattice with 9800 sites. The employed harmonic potential (5) has $V_{0}=-2.1, k=5.8$, and $r_{\max }=43 \sqrt{3}$.

optical lattice, as well as at its boundaries, can be directly measured with TOF images. To distinguish between edge and bulk currents we want to selectively obtain information about particular regions of the optical lattice. For this we can use TOF measurements following the release of atoms from within particular regions of the system. Such selective releases can be achieved by swapping the atoms within that region to a different internal state that does not experience the trapping potential. State-dependent manipulation such as this has previously been employed in optical lattice experiments to generate state-dependent tunneling among other effects $[26,27]$. As a result, the atoms in these regions freely expand and can be detected. This allows information, such as the atom density and velocity distribution, to be obtained. Releasing a small sample of lattice sites around the Haldane phase can therefore tell us about the physics of the edge and bulk currents.

We would like to investigate both the short-time and longtime TOF images. A short-time expansion of a sample of sites can already reveal the existence of the bulk and edge currents. In this case we take a horizontal sample of sites, as shown in Fig. 3(a), when the system is in a stationary state inside a harmonic trap. Then, we remove the trap and let the atoms inside that sample freely expand. After sufficiently small times a distinctive population pattern is formed. By measuring the relative inhomogeneities in the population after the expansion $\Delta_{y}(x, y ; t)=[n(x, y ; t)-n(x,-y ; t)] / n(x, y ; t)$ we observe that there are more particles at the top of the sample in the bulk of the Haldane phase region, while at its boundaries the particles accumulate at the bottom of the sample, as shown in Fig. 3(b). We measure the final population inhomogeneities in a smaller region than the original sample to avoid regions where the final density vanishes. These particle density differences point to the existence of a particle density 


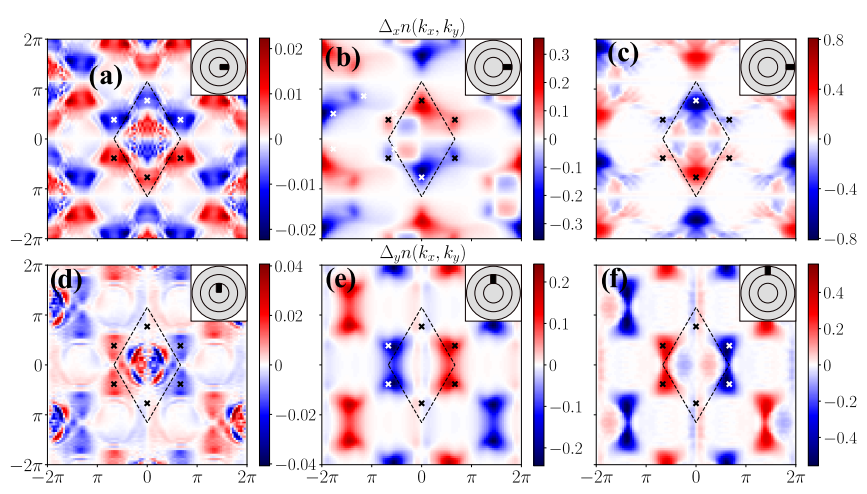

FIG. 4. Density differences in the TOF images taken at different phases of the system. The dotted lines mark the Brillouin zone of the hexagonal lattice and the crosses show the location of the Dirac points. Insets: Sketch of the rectangular region where particles were sampled for the TOF image. The circular coronas show the three topological phases in the system. (a)-(c) Relative difference $\Delta_{y} n\left(k_{x}, k_{y}\right)$ for samples taken along the $x$ axis of the lattice. Clearly there are currents moving along the vertical direction, with the bulk currents (b) traveling opposite to the edge ones (a), (c). (d)-(f) Relative difference $\Delta_{x} n\left(k_{x}, k_{y}\right)$ for samples taken along the $y$ axis of the lattice. There are currents moving along the horizontal direction, with the bulk currents traveling opposite to the edge ones. Data shown is for $T=0$ and a lattice with 9800 sites. The employed harmonic potential (5) has $V_{0}=-2.0, k=5.5$, and $r_{\max }=38 \sqrt{3}$.

current propagating perpendicular to the local gradient of the trap inside the topological phase in accordance with (2) and two counterpropagating currents at the topological phase boundaries as dictated by (1).

To obtain the signature of density currents from the long-time TOF images we select smaller regions of the lattice that isolate the bulk from the edge of the topological phase. Relative differences in the velocity distribution over the Brillouin zone for different regions of the trapped sample can signal a net flux of particles traveling in a certain direction. This is shown in Figs. 4(a)-4(c), where the colormap pictures the relative difference $\Delta_{y}\left(k_{x}, k_{y}\right)=$ $\left[n\left(k_{x}, k_{y}\right)-n\left(k_{x},-k_{y}\right)\right] / n\left(k_{x}, k_{y}\right)$ in the TOF sampling of rectangular patches along the $x$ axis of the optical lattice (see inset). A clear difference in the momentum population with opposite $k_{y}$ is seen, indicating the existence of currents moving along the $y$ direction of the lattice. Moreover, the direction of movement in (b) is opposite to that of (a) and (c), in agreement with Fig. 2. Figures 4(d)-4(f) show the relative density difference $\Delta_{x}\left(k_{x}, k_{y}\right)=\left[n\left(k_{x}, k_{y}\right)-n\left(-k_{x}, k_{y}\right)\right] / n\left(k_{x}, k_{y}\right)$ for samples along the $y$ axis of the lattice. In this case, currents flow along the $x$ axis, with the direction of the bulk currents (e) opposite to the direction of the edge ones (d), (f). The strength of the relative population imbalances is similar in the case of the bulk and outer edge currents [Figs. 4(b), 4(c) 4(e), and $4(\mathrm{f})]$ to those that have already been measured in several ultracold atom experiments [28,29].

To determine the behavior of the currents observed in TOF images under realistic conditions met in experiments we study the stability of the currents against temperature, local potential, and coupling disorder as well as variations in the strength of the harmonic potential. These effects are
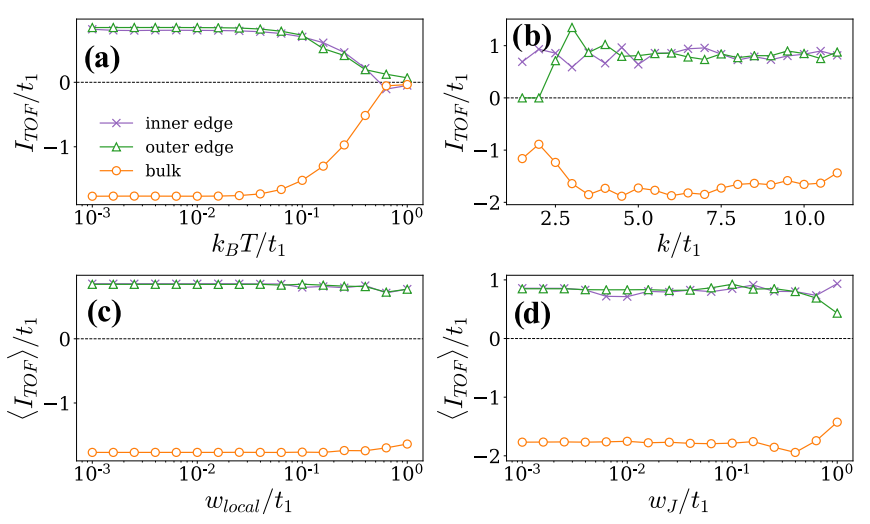

FIG. 5. (a) Stability of the edge and bulk TOF currents against the temperature of the optical lattice. Currents decay when the temperature reaches the energy gap of the Haldane model. (b) TOF currents when the trap strength $k / t_{1}$ is widened. For $k / t_{1}>2.5$ all three phases are present in the system and the edge and bulk currents are stable. (c), (d) Mean TOF currents over 15 samples with local (c) and coupling (d) disorder in the trap. The mean currents remain stable even when the scale of the disorder is comparable to the energy gap. Data shown is for $T=0$ and a lattice with 9800 sites. The harmonic potential (5) has $V_{0}=-2.0, k=5.5$, and $r_{\max }=38 \sqrt{3}$.

commonly present in optical lattice implementations. As the edge and bulk currents have a topological origin we expect them both to be largely resilient against these perturbations [10]. To proceed we define the net TOF current as

$$
\boldsymbol{I}_{\mathrm{TOF}}=\sum_{\boldsymbol{k} \in \mathrm{BZ}} \boldsymbol{k} n(\boldsymbol{k}),
$$

where we weight all the momenta inside the Brillouin zone with the density distribution $n(\boldsymbol{k})$ measured by the TOF images. We sample regions of the lattice as in Fig. 4 to distinguish between bulk and edge density currents. Figure 5(a) shows the behavior of the TOF currents (8) against the temperature of the system. We observe that the topological currents remain intact until the temperature is comparable to the energy gap of the Haldane model. Usual temperatures in cold atom experiments of the Haldane model are $\sim 0.2 T_{F}[19,26]$, within the region where we find edge and bulk currents to be stable. In Fig. 5(b) we test the stability of the currents against the strength of the trap potential (5) parametrized by $k / t_{1}$. For wide enough traps capable of supporting the three phases of the system, we observe stable TOF currents with intensity independent on the trap potential. In Fig. 5(c) we add a local disordered potential in the Hamiltonian with values randomly sampled from the uniform distribution $\left[-w_{\text {local }} / 2 t_{1}, w_{\text {local }} / 2 t_{1}\right]$, and in Fig. $5(\mathrm{~d})$ we multiply each coupling of the model with a value drawn from the uniform distribution $\left[1-w_{J} / 2 t_{1}, 1+w_{J} / 2 t_{1}\right]$ modeling imperfections in the designed Hamiltonian. In both cases we observe that the mean edge and bulk currents are resilient to all these forms of disorder. The disorder present in ultracold atom experiments in optical lattices is almost negligible, well within the region of stability of the bulk and edge currents.

Conclusions. To summarize, we have presented a general and versatile way to investigate the currents of a Chern 
insulator with TOF images. In particular, we focused on the behavior of the Haldane model simulated by ultracold atoms in optical lattices. In the presence of inhomogeneous potentials arising, e.g., by the harmonic trapping of the atoms topological bulk currents emerge along with the edge currents. The bulk currents are proportional to the gradient of the local potential, which naturally arises in the optical lattice experiment due to the harmonic trapping.

The topological origin of both the edge and bulk currents make them robust against perturbations, such as inhomogeneous potentials superimposed on top of the lattice, errors in the exact values of the couplings of the Haldane model, variations in the trapping potential of the atomic cloud as well as finite temperature. It also seems likely that the bulk currents are robust against fermion-fermion interactions, though we have not carried out a direct study. It was previously shown that the Chern number of the Haldane model remains invariant in the presence of both attractive and repulsive fermionic interactions, even for large interaction strengths [23]. Since the edge and bulk currents depend linearly on the Chern number we expect them to also be unaffected.
Bulk currents are also more versatile than edge currents and are easier to engineer and manipulate. More precisely, while edge currents require control over the shape of a topological sample, which must be done through a precise loading and hard potential walls, bulk currents are controlled by weak potential gradients, which are easier to engineer, both when created optically or by other electromagnetic fields. Hence, their implementation with optical lattices would open the way to employ them for quantum technologies $[14,15]$.

In compliance with EPSRC policy framework on research data, this publication is theoretical work that does not require supporting research data [30].

Acknowledgments. We would like to thank Monika Aidelsburger and Sofyan Iblisdir for inspiring conversations. This work was supported by the EPSRC Grant No. EP/R020612/1; Spanish Projects PGC2018-094792-B-I00 (MCIU/AEI/FEDER, EU), PGC2018-094180-B-I00 (MCIU /AEI/FEDER, EU), and FIS2015-63770-P (MINECO /FEDER, EU); CAM/FEDER Project No. S2018/TCS-4342 (QUITEMAD-CM), and CSIC Research Platform PTI-001.
[1] C. L. Kane and E. J. Mele, Phys. Rev. Lett. 95, 226801 (2005).

[2] C. L. Kane and E. J. Mele, Phys. Rev. Lett. 95, 146802 (2005).

[3] B. A. Bernevig and S.-C. Zhang, Phys. Rev. Lett. 96, 106802 (2006).

[4] M. Z. Hasan and C. L. Kane, Rev. Mod. Phys. 82, 3045 (2010).

[5] X.-L. Qi and S.-C. Zhang, Rev. Mod. Phys. 83, 1057 (2011).

[6] N. Goldman, G. Jotzu, M. Messer, F. Görg, R. Desbuquois, and T. Esslinger, Phys. Rev. A 94, 043611 (2016).

[7] Y. Hatsugai, Phys. Rev. Lett. 71, 3697 (1993).

[8] N. Hao, P. Zhang, Z. Wang, W. Zhang, and Y. Wang, Phys. Rev. B 78, 075438 (2008).

[9] E. Colomés and M. Franz, Phys. Rev. Lett. 120, 086603 (2018).

[10] C. N. Self, A. Rubio-García, J. J. García-Ripoll, and J. K. Pachos, Phys. Rev. B 102, 045424 (2020).

[11] Y. D. Lensky, J. C. W. Song, P. Samutpraphoot, and L. S. Levitov, Phys. Rev. Lett. 114, 256601 (2015).

[12] M. R. Geller and G. Vignale, Phys. Rev. B 50, 11714 (1994).

[13] S. Mondal, D. Sen, K. Sengupta, and R. Shankar, Phys. Rev. Lett. 104, 046403 (2010).

[14] S. Zhang and X. Zhang, Electrical and optical devices incorporating topological materials including topological insulators, U.S. Patent No. 9,024,415 (2015).

[15] S. K. Banerjee, I. L. F. Register, A. MacDonald, B. R. Sahu, P. Jadaun, and J. Chang, Topological insulator-based field-effect transistor, U.S. Patent No. 8,629,427 (2014).

[16] B. Scharf, A. Matos-Abiague, J. E. Han, E. M. Hankiewicz, and I. Žutić, Phys. Rev. Lett. 117, 166806 (2016).
[17] Y. Tanaka, T. Yokoyama, and N. Nagaosa, Phys. Rev. Lett. 103, 107002 (2009).

[18] L. Tarruell, D. Greif, T. Uehlinger, G. Jotzu, and T. Esslinger, Nature (London) 483, 302 (2012).

[19] G. Jotzu, M. Messer, R. Desbuquois, M. Lebrat, T. Uehlinger, D. Greif, and T. Esslinger, Nature (London) 515, 237 (2014).

[20] N. Goldman, J. C. Budich, and P. Zoller, Nat. Phys. 12, 639 (2016).

[21] N. Fläschner, B. S. Rem, M. Tarnowski, D. Vogel, D.-S. Lühmann, K. Sengstock, and C. Weitenberg, Science 352, 1091 (2016).

[22] L. Asteria, D. T. Tran, T. Ozawa, M. Tarnowski, B. S. Rem, N. Fläschner, K. Sengstock, N. Goldman, and C. Weitenberg, Nat. Phys. 15, 449 (2019).

[23] E. Alba, X. Fernandez-Gonzalvo, J. Mur-Petit, J. K. Pachos, and J. J. Garcia-Ripoll, Phys. Rev. Lett. 107, 235301 (2011).

[24] M. Buchhold, D. Cocks, and W. Hofstetter, Phys. Rev. A 85, 063614 (2012).

[25] F. D. M. Haldane, Phys. Rev. Lett. 61, 2015 (1988).

[26] G. Jotzu, M. Messer, F. Görg, D. Greif, R. Desbuquois, and T. Esslinger, Phys. Rev. Lett. 115, 073002 (2015).

[27] L. Riegger, N. Darkwah Oppong, M. Höfer, D. R. Fernandes, I. Bloch, and S. Fölling, Phys. Rev. Lett. 120, 143601 (2018).

[28] J.-P. Brantut, J. Meineke, D. Stadler, S. Krinner, and T. Esslinger, Science 337, 1069 (2012).

[29] X. Guo, W. Zhang, Z. Li, H. Shui, X. Chen, and X. Zhou, Opt. Express 27, 27786 (2019).

[30] https://epsrc.ukri.org/about/standards/researchdata/. 